Chapter 10

\title{
Breaking Through the Speed Barrier - Advancements in High-Speed Imaging
}

\author{
G.P.P. Gunarathne \\ Additional information is available at the end of the chapter \\ http://dx.doi.org/10.5772/56378
}

\section{Introduction}

\subsection{Origin, expansion and applications of ultrasonic imaging}

History and the discoveries of the use of ultrasound can be traced back to late $18^{\text {th }}$ century, but one of the major steps toward the practical use of ultrasound may be attributed to Lewis Nixon who invented the very first sonar type listening device in 1906 as a way of detecting icebergs [1]. Rapid developments of the use of ultrasound occurred since then, particularly after world war II; initially for underwater (sonar) and industrial uses, followed by developments for medical applications [2,3]. Today, the technology is widespread in Medicine, Non Destructive Testing (NDT) and Sonar in many specialised areas such as: industrial and medical imaging, study and classification of the properties of material and biological tissues, seismic explorations high-intensity applications etc. and the applications are growing. These rapid advances are directly related to the parallel advancements in electronics, computing, and transducer technology together with sophisticated signal processing techniques.

Irrespective of the field of applications, arguably one of the most important applications of ultrasound is "Imaging", which is the chosen subject of this chapter. However, it is important to note that imaging as applied to the three main application areas mentioned above; namely, NDT, Sonar and Medicine have fundamental similarities and also differences. For example, the speed of sound in water in the case of sonar and biological tissues are comparable $(\sim 1450 \mathrm{~m} /$ s) but sonar is extremely long range.

In the case of NDT, the speeds of sound in industrial materials are generally very much higher, although the penetration distances are comparable to that for medical imaging. On the other hand, sonar and medical imaging primarily relies on one form of ultrasound propagation, namely, compressional waves while in solid material, there are multiple propagational modes, 
such as compressional, shear, surface, creep, lamb and torsional waves etc. These different modalities have widely different characteristics and they can coexist with possible mode conversions depending on the particular geometry and test scenario. In NDT, the co-existence or co-generation of this multimode propagation can be both advantages in some situations, and equally become a nuisance in other cases.

In processing and assessing an ultrasound image, one of the most striking differences between industrial NDT and medical imaging is that in the case of the latter, there is good preanatomical knowledge of the part of the anatomy being examined; often with the possibility of supplementary data obtained from other forms of imaging such as MRI, CT scans etc. However, for NDT inspection, the target features (shapes, sizes and orientation etc.) are almost always largely unknown, thus the interpretation is largely based on the reliability of the images being produced. This is a major challenge in industrial imaging applications. On the positive side, the targets being imaged in NDT applications e.g. a crack in a structure generally tends to appear as a good acoustic discontinuity yielding good Signal-to-noise ratio (SNR) compared to that from a tissue boundary, since the latter is dependent on small impedance contrast.

\subsubsection{Dynamic range considerations}

For all imaging applications, the signal dynamic range is an extremely important consideration. Usually this could be very high; far above that may be accommodated by display equipment. For medical Imaging, the dynamic range of signals could be of the order of order of $100 \mathrm{~dB}$ covering both backscattered signals from tissues and specular reflections. (However, the range of interest is of the order of 40 to $50 \mathrm{~dB}$, which is the range covered by backscattered signals). Although impedance contrast is generally high in NDT and sonar, dynamic range of signals can still be very large depending on the application, not necessarily because of high attenuation as in the case of biological tissues but because of the size of the targets such as micro-defects in NDT or beam divergence in the case of sonar. In the case of sonar, the range is extreme and the signals of interest could be of the order of several hundred millivolts to submicro volts. The dynamic range can be evaluated for homogeneous media, such as water, by considering signal loss between targets of same strength placed at different axial distances $\left(R_{1} \& R_{2}\right)$ form equation 1 below, which helps formulating compensating strategies.

$$
\operatorname{Loss}(d B)=20 \log \frac{R_{2}}{R_{1}}+2 \propto\left(R_{2}-R_{1}\right)
$$

Where $\propto$ is the attenuation coefficient of the medium at the frequency of interest. Accommodating large dynamic range and depth gain compensation are therefore features common to all imaging systems; albeit for different reasons. Once the ranges and the loss characteristics are known, accommodation can be handled electronically, e.g. by using logarithmic compression and Time-varying-gain (TVG) functions to accommodate the signals within the much limited display dynamic range.

In order to understand the potential of imaging and areas of improvement, it is necessary to examine the fundamental characteristics of common imaging modalities, which is the subject 
of the following section. The discussion does not include a comprehensive treatment of imaging techniques in use, but the ones that represent properties central to the understanding of primary limitations.

\subsection{General principles}

The simplest form of ultrasonic visualisation is what is known as the A-scan. Here an ultrasonic transducer generates a short pulse of high frequency sound which is coupled to the test medium. Echoes generated from any acoustic discontinuities within the path of the ultrasound beam are received, usually by the same transducer, and are displayed on a screen or a monitor as intensity modulated signals. The technique essentially provides (a) Target-depth information (b) An indication of the extent of the discontinuity and to a much lesser extent information on its orientation or shape. Since the time required for displaying the echoes is virtually equal to the time of flight of the acoustic pulse within the object medium, it gives the maximum temporal resolution for moving targets. However, the interpretation and information obtained from an A-scan (1- spatial dimension) or that derived from it is very limited, highly operatordependent and therefore of limited use in diagnostic applications.

However, a collection of A-scans in a given plane in the test object could provide a twodimensional map (or an image) of the acoustic discontinuities. This requires acquisition, storage and display of successive A-scan lines requiring digital data storage and processing. These line-serial scans may be generated in a number of ways: mechanically moving the transducer along a given direction; using an array of transducers which are switched on in succession to mimic a mechanical movement (linear or curvilinear array scanning). Images thus generated represent a two-dimensional (2-D) view of the target medium and are commonly known as B-scans.

Alternatively, the ultrasound beam may be electronically steered using a transducer array (phased array scanning) or by a mechanically rotating transducer. The 2-D images thus obtained are normally referred to as sector B-scans.

\subsubsection{Real-time versus determinism}

One of the confusing terminologies in imaging (or any other form of data presentation) is "Realtime". The naturally implied meaning of the term real-time is: "as it happens". This meaning is incompatible with laws of Physics since nothing can be observed at the same instant as something happens. So, image presentation can only approach this ideal of real-time, provided that what an observer sees in an image is close enough to what was happening to the object as a whole, both in time and spatial resemblance. Then the expression "real-time" can be considered appropriate. For example, an observer seeing a trajectory of a meteoroid views this in real-time because the velocity of light is at least 6 to 7 orders of magnitude higher than the moving object. But this is not the case, for example, seeing a moving heart valve in an ultrasound image taking a few milliseconds to form just one frame. Obviously, the image is temporally and spatially distorted. Therefore a target that appears to be moving in a succession of image frames is not seen "as it happens" and therefore not true real-time. The term "Pseudo 
Real-time" is a more meaningful definition in such cases where the image is seen to be live, while the velocity ratio (i.e. the speed of the target to that of sound) is significant, or forming an image frame takes significant time.

On the other hand, "Determinism" can be an important concept in defining a key aspect of image integrity. This can be a predominant requirement - for example in quantitative image analysis where accurate spatial information is important. The use of the term "determinism" in this context does not really mean "as it happens" (although the time interval between a moving target and its presentation as an image is usually small). Determinism essentially means that the spatial integrity of a moving target is persevered in the image to a high degree. In the case of ultrasound the theoretical limit of spatial determinism can only be reached if a single ultrasound pulse emitted by an imaging device produces a complete image field of the object within its time of flight. This is clearly not achievable with the existing line-serial imaging technology, but it is one of the main features of the new hybrid imaging system described later in this chapter.

\subsection{State-of-the-art techniques}

In late 1980's, the techniques for generating 3-D images from a collection of 2-D images have been demonstrated [4]. Producing a single frame of such an image may have taken about 20 minutes then, but with the advancement of computing power and processing techniques, live 3-D and 4-D volume imaging reaching pseudo-real time volumes of surface features have now come into existence $[4,5]$.

One of the fairly recent additions to B-mode imaging is the so called Zone-sonography [6]. A main feature in this system is the acquisition of data from a relatively low number of zone sectors, as opposed to line-by-line acquisition, thereby significantly improving the temporal artefacts inherent with the conventional methods. It has been claimed that in some cases, this approach could produce speed improvements of up to 10 times compared to conventional lineserial imaging systems [6], although the zones in themselves are produced using serial scanning.

In addition, in the case of medical imaging, various echo enhancement techniques such as Coded Excitation (CE) and Digitally Encoded Ultrasound (DEU) which improve sensitivity, penetration and contrast are now been widely used [7, 8]. CE is a pulse compression technique which is designed to differentiate and boost weak return signals from deep within the body. This is done by transmitting an encoded pulse sequence, isolating the coded return signal and amplifying only this signal, while regaining longitudinal resolution which is distributed within the insonifying pulse train [7]. Some systems using CE can also show blood flow together with the body tissue as a B-mode image, without the need for overlay as would be the case with Doppler imaging. Tissue Harmonic Imaging (THI) is another widely used technique which reduces haze, clutter and image artefacts. In this approach, higher harmonic components of the echoes generated by the tissues due to non-linear propagation are used instead of the fundamental [9]. 
It is also important to note that there has been renewed interest in some of the early developments that uses direct imaging technology such as ultrasonic cameras and other imaging modalities [10, 11].

\section{Limitations of the conventional technology}

\subsection{Basic limitations}

A fundamental limitation with all of the above existing technology, which arises from the need for line-serial scanning, is the loss of temporal resolution. Attempts to improve this aspect by increasing computing power alone cannot give the full-potential of real-time imaging and may only give improvements with largely diminishing returns.

The second limitation, for example when using linear-array technology, is the use of smaller effective aperture compared to the size of the total array aperture, hence affecting the lateral resolution achievable. These two limitations are analysed below in order to reveal the extent to which they affect potential performance in ultrasonic imaging. Although the discussion is based on using linear arrays for clarity, the same considerations are valid for other configurations.

Figure 1 below shows a linear array transducer coupled to a test medium. The test object is assumed to be homogeneous and have a depth $(\mathrm{d})$. The array consists of $(\mathrm{N})$ elements with a total aperture size (A). The elements are usually switched on as a group (as shown) rather than single elements to improve lateral resolution, since this depends on the effective aperture, as depicted in equation 3 . The next group in the firing sequence advance by only one element thus keeping the line resolution equal to the spacing of the array elements.

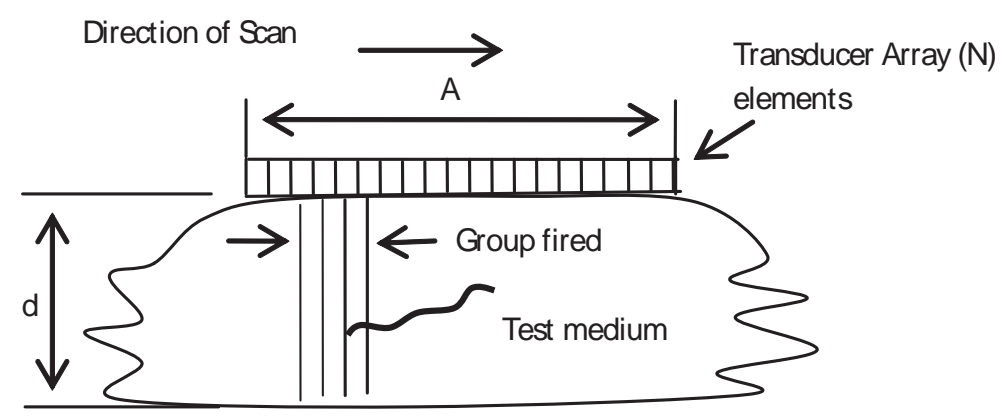

Figure 1. Line-serial scanning

Between individual scanning lines, a rest period $\left(t_{\text {rest }}\right)$ is applied before firing the second consecutive group of elements as a practical requirement to allow multiple echoes to die down and time for acquisition, storage and display of data. 


$$
\begin{aligned}
& \mathrm{t}_{\mathrm{f}}=\left(\mathrm{N}-\mathrm{N}_{\mathrm{G}}\right)\left[\left(\frac{2 \mathrm{~d}}{\mathrm{c}}\right)+\mathrm{t}_{\text {rest }}\right] \\
& \therefore \text { Frame rate } \approx \frac{1}{\mathrm{t}_{\mathrm{f}}}
\end{aligned}
$$

With reference to Figure 1, time $\left(t_{\mathrm{f}}\right)$ taken to produce one frame is:

where, $\mathrm{N}_{\mathrm{G}}$ is the number of elements in the group firing at any one time and $\mathrm{c}$ is the speed of sound. Hence, as an example, when using an array with 150 elements, coupled to a medium in which the velocity of sound is $1.54 \mathrm{~mm} / \mu \mathrm{s}$, thickness $20 \mathrm{~cm}, \mathrm{~N}_{\mathrm{G}}=50$, and neglecting $\mathrm{t}_{\text {rest }}$, it can be seen that the maximum frame-rate achievable is about 75 . This will be even less when $\mathrm{t}_{\text {rest }}$ is applied.

However, the above frame rate may sound adequate, and indeed so for many cases, but the line-serial scanning introduces a basic limitation in the case of fast moving targets such as heart valves or machinery. During the scanning time within a frame, one part of the target may have displaced significantly relative to another, causing spatial distortion, thus a frozen image of the target at any time may differ significantly from its actual position or shape. This is illustrated in Figure 2.

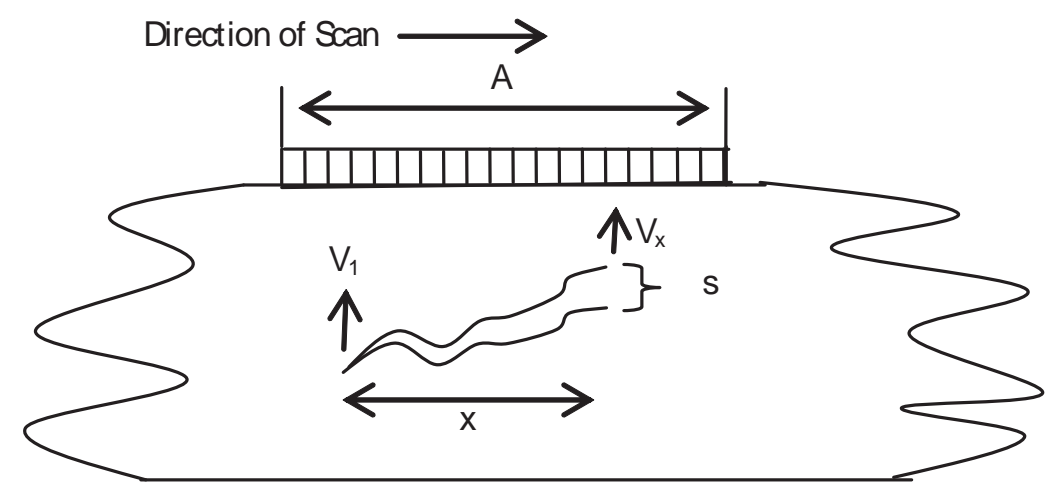

Figure 2. Formation of temporal artefacts

With reference to Figure 2, spatial distortion $(\Delta s)$ could be written as:

$$
\Delta \mathrm{s}_{(\mathrm{x})}=\frac{\mathrm{x}}{\mathrm{A}} \times \mathrm{t}_{\mathrm{f}} \times \mathrm{v}_{\mathrm{x}}
$$

where, $\mathrm{v}_{\mathrm{X}}$ is the velocity of a point $(\mathrm{x})$ on target. 
The other limitation with a linear array scanner, as mentioned above, is the degradation of lateral resolution arising from the size of the aperture formed by the group of elements firing at any instant of time. The lateral resolution is limited by the beam width of the transducer or the group of elements. The diffraction limited beam width for a given transducer of aperture (D) could be expressed as:

$$
\delta \theta=\frac{1.22 \lambda}{\mathrm{D}}
$$

where, $\lambda$ is the wavelength of sound in the medium. As can be clearly seen, for each scan line, $\mathrm{D}$ is determined by the number of elements within the group fired and this is clearly very much less than the total aperture size (A) of the array. Hence, the lateral resolution corresponding to that achievable with the fully available aperture is not realised.

\subsection{Ideal properties of an imaging system}

From the above discussion, it is clearly evident that two key properties of an ideal real-time ultrasonic imaging system is that it should be able to produce a complete image frame of the object volume from one insonifying pulse, while utilizing the full aperture available for each frame of the image. Additionally, an ideal system should produce focused images of the whole object field at the same instant of time irrespective of the distances of targets from the surface (Isochronicity) while maintaining accurate object-to-image spatial resemblance (image linearity). It is also clear that the existing technology utilising line-serial scanning cannot achieve these ideal properties and therefore alternative techniques may be needed to improve speed and high resolution capabilities beyond that feasible with conventional technology. This requires investigating non-conventional imaging modalities that can possess those capabilities, and the ways of overcoming limitations that may have precluded their use in practice. The next section presents some early developments that have some of the above key properties achieved through direct ultrasonic image reconstruction. Beyond academic interest, these techniques have not realised in large-scale use except in very specific applications due to other inherent problems.

\section{Non-conventional methods}

\subsection{Direct ultrasonic imaging}

Direct ultrasonic image visualization using alternative methods, such as ultrasonic holography, Bragg diffraction imaging etc. have been documented by many researchers in the past $[12,13,14]$. One such technique that has the above ideal properties is the Direct Ultrasonic Visualization of Defects (DUVD) system demonstrated by Hansted in the 1970's [12]. As schematically shown in Figure 3(a), this is a passive acousto-optical configuration which uses a pair of ultrasonic lenses with a common focus to form an ultrasonic image of the object field in a transparent medium from echoes received from a test object; much in the same way as an 


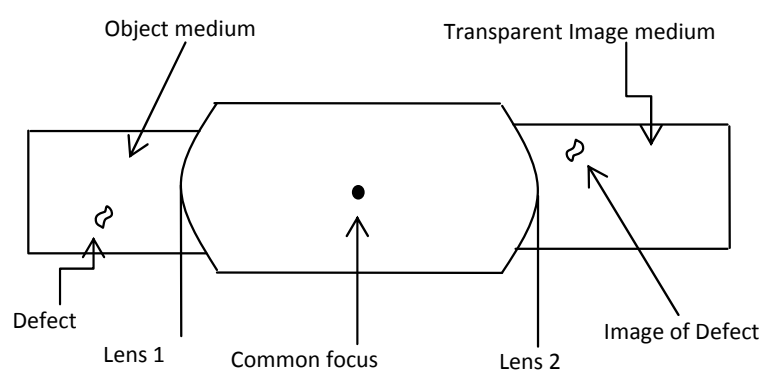

(a)

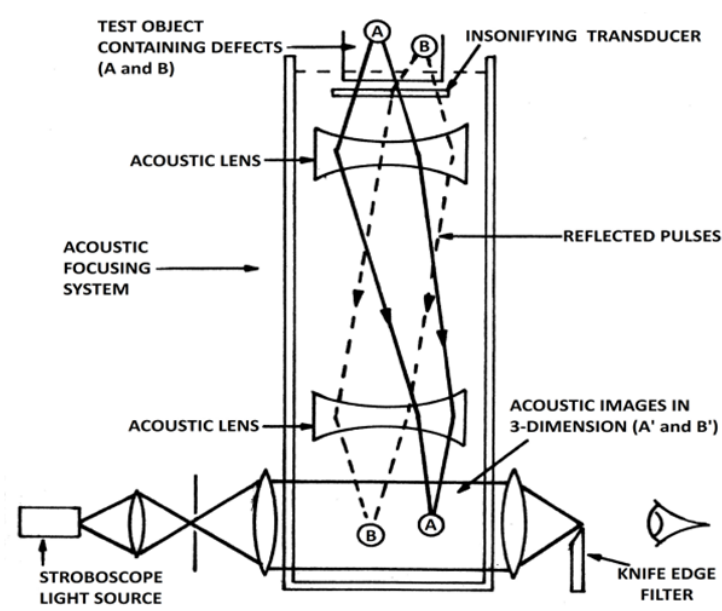

(b)

Figure 3. Direct Ultrasonic Imaging (DUVD) (a) DUVD Basic configuration; (b) A liquid coupled version of DUVD

optical lens producing an optical image. The insonifying transducer is physically bonded or intimately coupled via a liquid medium to the test object as part of Lens 1 . Returned echoes are brought into focus by the acoustic lens arrangement in the image medium. The ultrasonic images thus produced is made visible by stroboscopic light which is synchronized to the transmitted insonifying ultrasound pulse, but with a fixed delay to allow the image to be formed and viewed at the point of best focus.

One of the DUVD realisations with liquid coupled lenses and a schlieren acoustooptical visualisation system developed by Hansted [12] is shown in Figure 3(b) above. It is interesting to note that the DUVD has many ideal features: It operates in Real-time; producing 3D images of the complete object field with every single insonifying pulse. 
However, two main problems with the DUVD system shown above are: very low sensitivity and its design is such that the test objects virtually becomes part of the system; thus severely limiting flexibility as can be seen from the block diagram representation of the DUVD below (Figure 4).

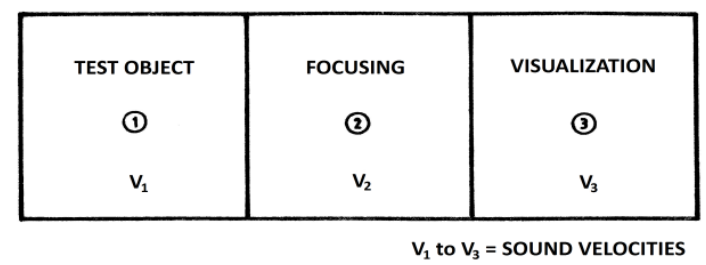

Figure 4. DUVD block schematic (Passive system). The insonifying transducer is bonded at the boundary between medium $1 \& 2$.

Further attempts to improve this approach have been reported by others in the late 1970's [13, 14]. But these were also passive systems and apart from theoretical interest the performance was practically inadequate.

\subsection{A key development in the 1980's}

\subsubsection{Conceptual development}

Because of the attractive features of direct ultrasonic imaging without the need for line serial scanning, a significant development was undertaken in the mid 1980's [15]. For initial feasibility studies, an active 2D version of the DUVD concept was considered. A major advancement was the introduction of amplification between a set of transmitting and receiving arrays of transducers; thereby solving the problems of low sensitivity and inflexibility inherent with the DUVD approach. This decouples the test object from the rest of the system as shown in Figure 5 below.

Since the system is now transformed from passive sonoptics to an active sampling and reconstruction technique, the design specifications were derived by detailed computer simulations and practical investigations to achieve satisfactory image quality. It should be emphasised that the requirements for image reconstruction for this system is very different to conventional imaging. It essentially involves image formation utilizing amplitude and phase of signals as represented by equation 5 and Figure 6 below.

$$
P_{(x, y)}=\sum_{i=1}^{N} a_{i} \frac{\exp ^{-j\left(k r_{i}+\varnothing_{i}\right)}}{r_{i}}
$$

where, $\mathrm{p}(\mathrm{x}, \mathrm{y})$ represent the acoustic pressure at a point $\mathrm{x}, \mathrm{y}$ in the image space as in Figure 6 below, $a_{i}$ is the normalised signal amplitude, $r_{i}$ is the distance to the point $(x, y)$ from the $i^{\text {th }}$ element, $\varphi_{\mathrm{i}}$ is the relative phase of the $\mathrm{i}^{\text {th }}$ element, and $\mathrm{k}$ is the wave number. 


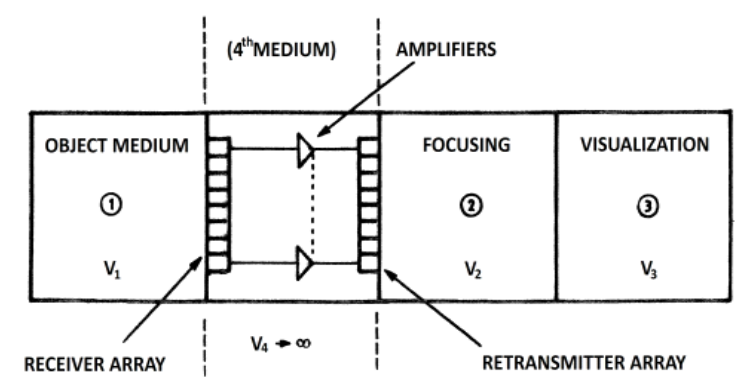

(a)

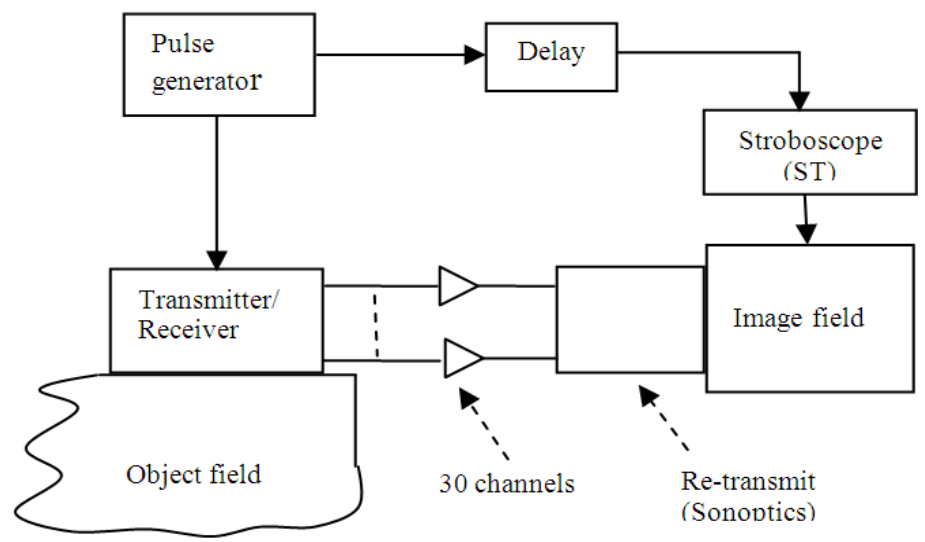

(b)

Figure 5. (a) - Active system block diagram with arrays (b) - Active system schematic block diagram

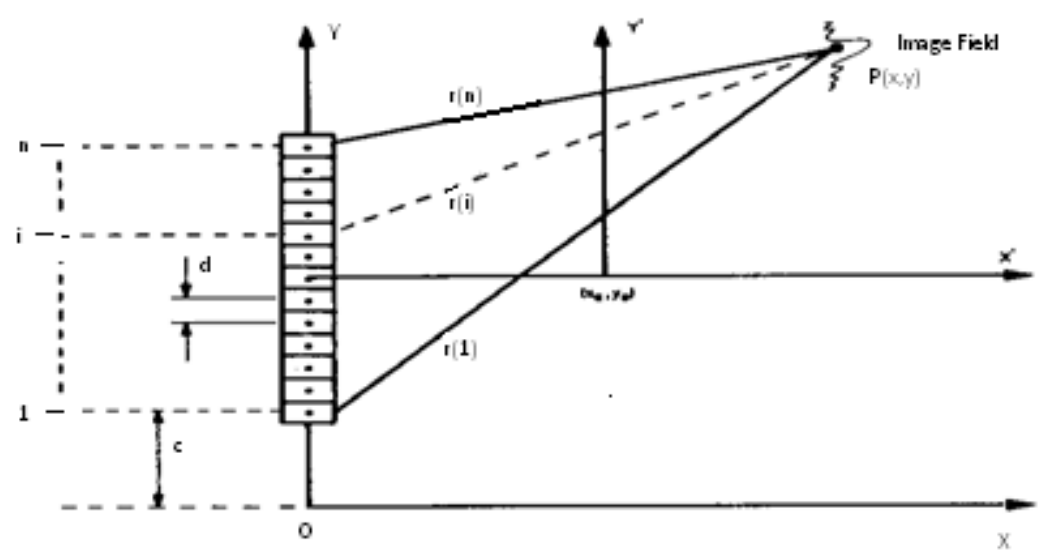

Figure 6. 
Therefore in order to achieve good image quality, stringent control on the uniformity of the transducer elements in terms of their amplitudes and phase responses were essential requiring special fabrication techniques, since achieving close elemental uniformity in transducer arrays is a challenging task.

The details of the prototype design while achieving the ideal features described in section 4.1 above namely: linearity, isochronicity and maximum lateral resolution corresponding to the total acoustic aperture are presented in the Appendix.

\subsubsection{First prototype system}

For the feasibility study of the active direct imaging concept, a 2-D version of the 3-D DUVD sonoptics was chosen. However, it is important to note that as for the 3-D sonoptics, the same acoustooptical relationships could be maintained by using cylindrical lenses to give an image field similar to a B-scan. Because of the flexibility introduced by the sampling and retransmitting acoustic arrays, the operation of the lenses could be emulated by a number of different ways; e.g. by electronic focusing to represent both lenses, using solid cylindrical lenses, or by a combination of both. It should be emphasized that once the lens characteristics required are implemented, there is no further special requirement for dynamic focusing as the entire image field will be in focus at one instant of time requiring only one excitation pulse to produce the image of the whole object field.

After investigating different possibilities, the following configuration shown in Figure 7 below was used for the first prototype.

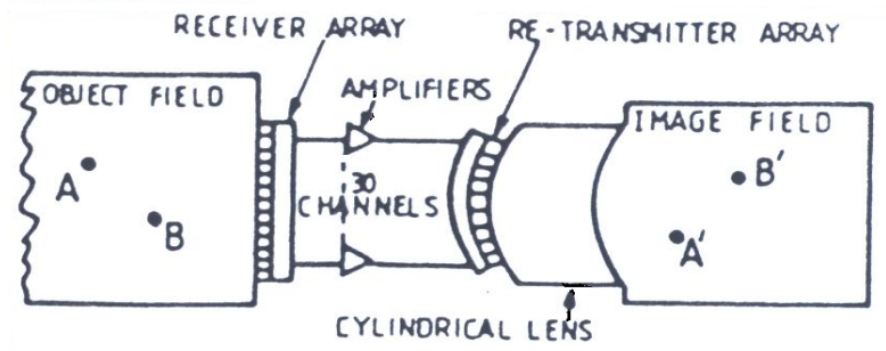

Figure 7. Active system configuration with conversion of DUVD sonoptics to maintain image linearity, Isochronicity and resolution.

Figure 8 below shows the construction of the retransmitting section of the system with retransmitting array, cylindrical lens and image medium. The design parameters were chosen to image a section of steel of depth up to $40 \mathrm{~cm}$, which is well in excess of typical applications.

Figure 9 show the trolley-mounted $1^{\text {st }}$ prototype system designed in accordance with the simplified block diagram shown in Figure 5. The optical assembly is equipped with an ultrashort stroboscopic light source [16] mounted on the right and a video camera for capturing 


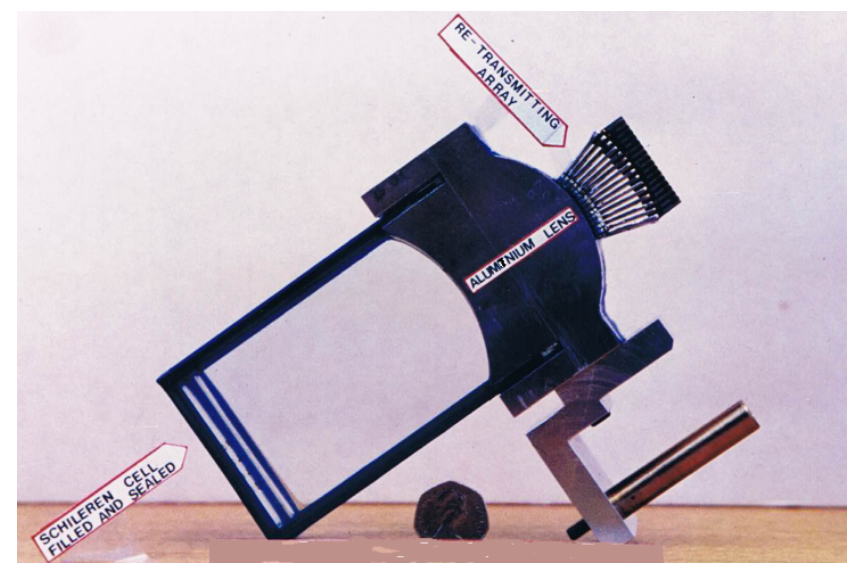

Figure 8. Sonoptical assembly including the imaging cell

images on the left along the optical axis. For experimentation, only 15 of the 30 available channels were used in this prototype.

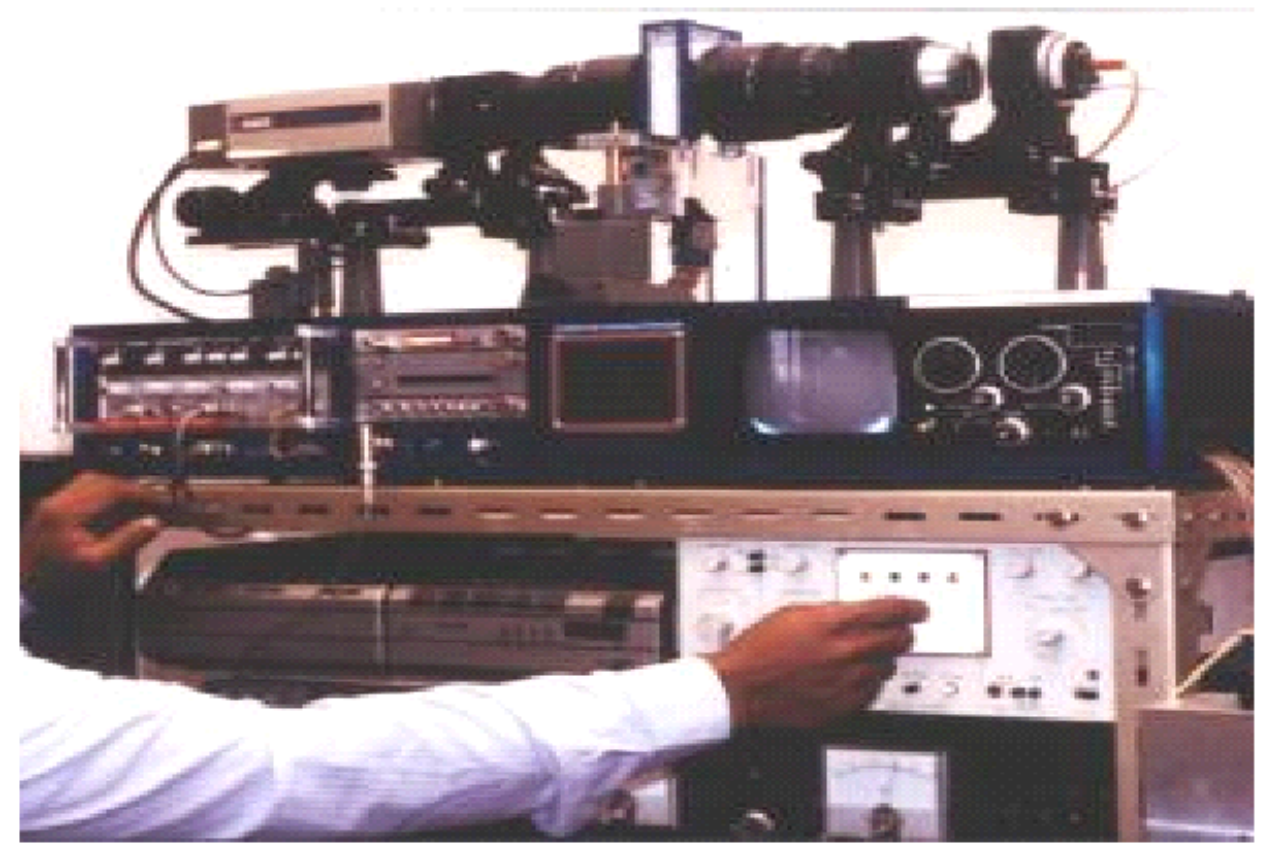

Figure 9. The active acoustooptical imaging system ( $1^{\text {st }}$ prototype) 


\subsubsection{System operation}

When testing, the receiving probe array is coupled to the test object medium with a liquid couplant such as oil or jelly. The test object is insonified with a short pulse of ultrasound and the delay of the stroboscopic light source is set appropriately such that the acoustic images are optically frozen in time within the optical imaging medium (schlieren cell) at the instant of best focus.

The resulting optical image of the whole object field can be seen either by naked eye or can be captured with a video camera. Since the time required to form a complete image field is now only limited by the time of flight of the acoustic signals the frame rate could reach the theoretically possible maximum limits (e.g. in excess of 1000 frames per second).

\subsubsection{Results from the first prototype}

\section{Image linearity and Isochronicity}

Although the system was designed with 30 channels, only 15 were used because of the difficulty of making elements of the arrays with sufficiently close characteristics as required for this imaging topology. However, as can be seen from the images, they are exceptionally of high quality in terms of image clarity and resolution. Figure 10 shows a test block with side drilled holes and the resulting image, clearly demonstrating image linearity and isochronicity as expected from the system design.
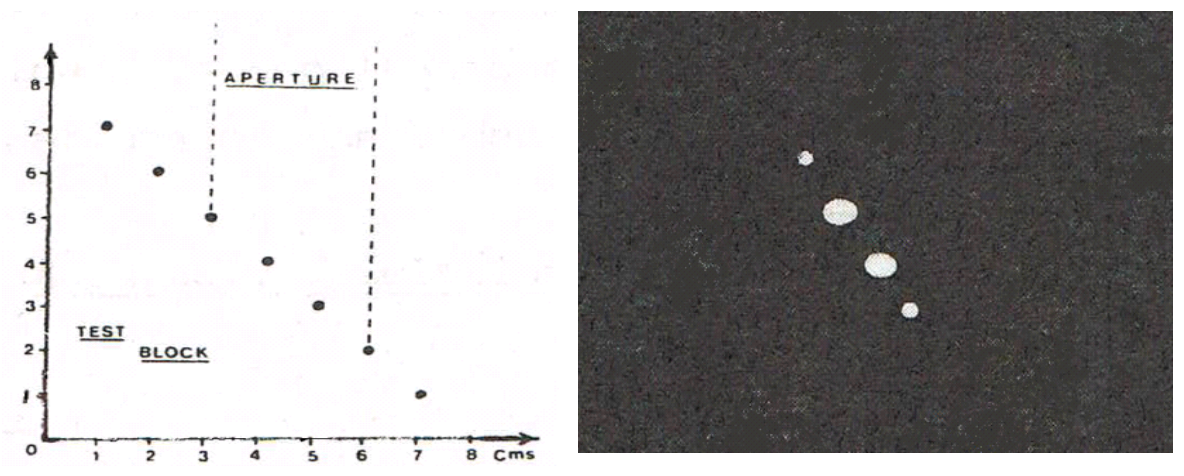

Figure 10. Images of $2 \mathrm{~mm}$ side drilled holes.

Notice the axial magnification as depicted in equation 10 in appendix. The targets central to the array obviously have stronger return echoes, so they are somewhat saturated due to relatively low dynamic range of the channel amplifiers used. Figure 11 below show a test object, again with side drilled holes and the respective image with lateral and axial magnifications equalised. This image demonstrates exceptional image quality with object-to-image spatial relationship correctly maintained. 

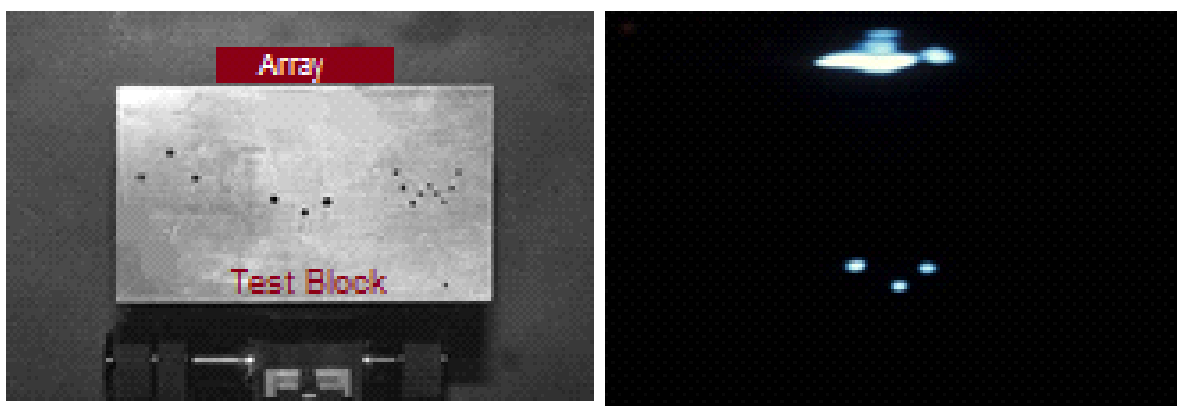

Figure 11. Imaging side-drilled holes in a test block

Figure 12 below shows an actual T-weld being tested with the prototype system and the image of a crack in the weld. This micro-crack was actually visible from the ground side surface of the test block.
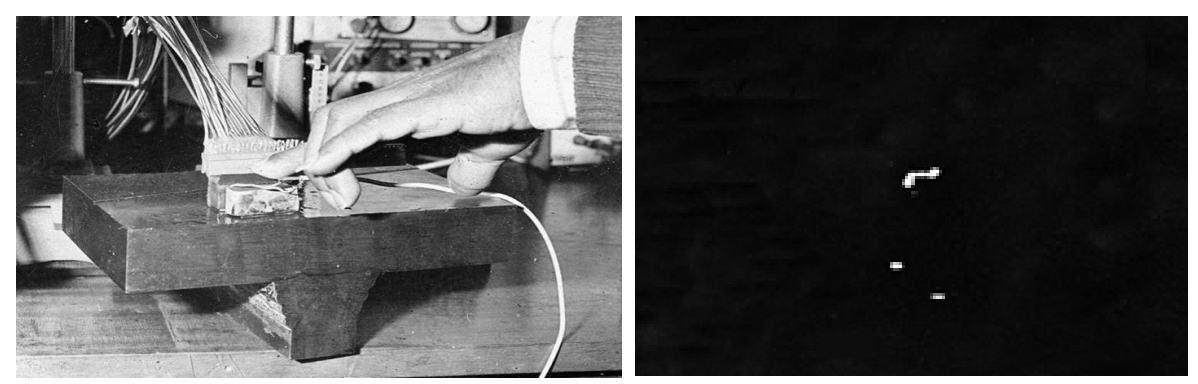

Figure 12. Image of a micro crack in a T-weld

\subsubsection{Practical limitations of the first prototype}

The results from the first prototype have clearly demonstrated the potential of direct ultrasonic imaging reaching performance close to theoretical limits. These include: maximum possible speed of imaging, maximum lateral resolution for the size of the arrays used, forming focused images of the whole object field covered by the transducer aperture.

However, there were still practical limitations. This system also had a small field of view approximately that covered laterally by the array aperture; in this case $\sim 3 \mathrm{~cm}$ wide. This is too 
narrow in practice since wider coverage requires moving the receiving array over the test surfaces, which are typically uneven. Since the image reconstruction is heavily dependent on the amplitude and phase information of the signals, unevenness influences the image quality to a greater extent than conventional imaging methods; although in ideal conditions, excellent results could be achieved as shown in the results section above.

\section{Advancements in non-conventional methods - Development of a high- speed, computer-controlled hybrid scanner}

From the performance of the acousto-optical imaging system shown in Figure 9, it was evident that to meet practical requirements and to advance the potential benefits, the following characteristics would be very desirable.

1. Ability to scan a test object for off-axis imaging, thus overcoming the limitation of small field of view.

2. Dynamic focusing along specified paths and sites to improve image quality.

3. Means of providing a degree of image enhancement.

The first property adds a much greater degree of freedom to test uneven surfaces from selected or prepared locations. The resulting images would be similar to a B-mode sector scan, but the imaging modality is such that it produces image zones for required sectors as opposed to individual line serial scanning, with just a few overlapping sectors enabling much higher frame rates to be achieved. Since there is no requirement for focusing in the image medium, as that is already taken care of by the sonoptical design, the scanning simply means that only the insonifying beam need to be steered to illuminate the object sector.

Since the image reconstruction is implemented with a sonoptical system designed with paraxial ray equations, when imaging a wide field like a sector, there is likely to be a significant degree of peripheral geometrical aberration in the image field. It should be possible to correct these dynamically, since for each image zone, implementation of an appropriately precalculated delay in the firing of the stroboscopic light source, channel gain/phase manipulation is possible.

Inclusion of the above properties is the basis of the development [17-19] described below. Figure 13 shows the basic block diagram of the hybrid prototype developed, incorporating scanning hardware (SCH) controlled by a microcomputer (PC). Dynamic control hardware $(\mathrm{DCH})$ is intended to provide gain equalisation during off-axis imaging and time varying gain (TVG) to produce a uniform image field. Field Focus Control (FFC) ensures that the stroboscopic illumination is synchronised such that the image field is optically frozen at the instant of best focus. It is also intended to provide a degree of off-axis aberration correction with sectordependent focusing delay control. 


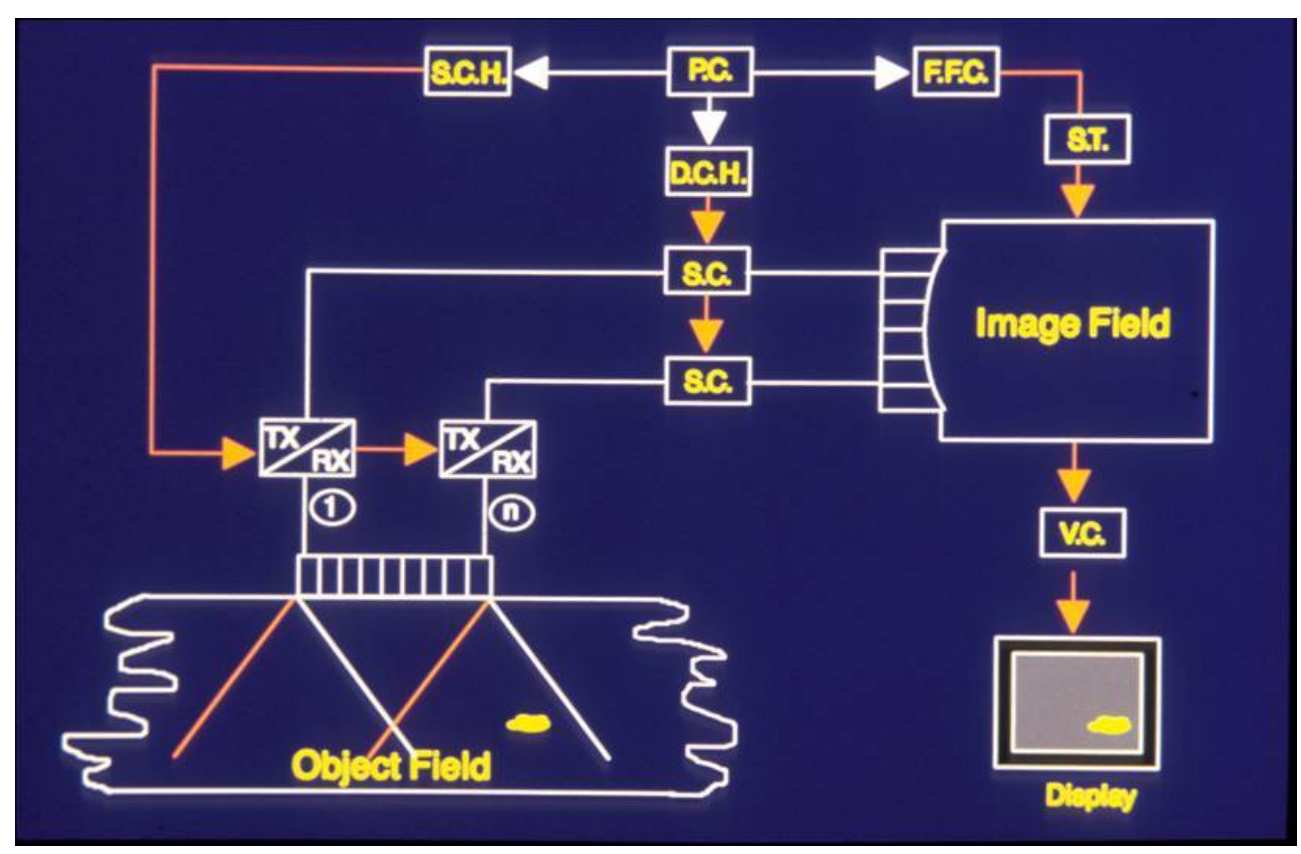

Figure 13. Basic block diagram of the hybrid scanner

\subsection{Principle of operation}

As mentioned previously, in contrast to conventional imaging, this system also operates on the basis of forming an ultrasonic map of the object field from received echoes inside a visible medium. Once an ultrasonic pulse illuminated the object medium, the acoustic signals intercepted by the receiving array is retransmitted into the image medium through the acoustic focusing system after conditioning and boosting signal power using signal conditioning hardware (SC). The re-transmitting section consists of an identical set of transducer array (except for the shape) to that of the receiving array, coupled to a specially designed ultrasonic focusing arrangement according to the requirements described in 8.1.1 (Appendix). Two important characteristics of this focusing lens design are that all the target echoes are brought into focus at the same instant of time irrespective of the target depth while maintaining image spatial linearity, so that a complete image frame is produced by just one insonifying pulse in real-time.

When the acoustic image field is at the time of best focus, an ultra-short stroboscopic light pulse of the order of a few nanoseconds is emitted, which produces a visible image. This can be viewed directly or captured by a video camera. Since the maximum repetition rate is now only determined by the time-of-flight of the sound pulse, the system achieves the highest speed of operation theoretically possible in ultrasonic imaging, eliminating the problem of temporal artefacts inherent with the existing systems. 
Figure 14 shows a more detailed block diagram of the improved design with a possible use in testing of welds. Figure 15 shows the first prototype hybrid scanner developed.

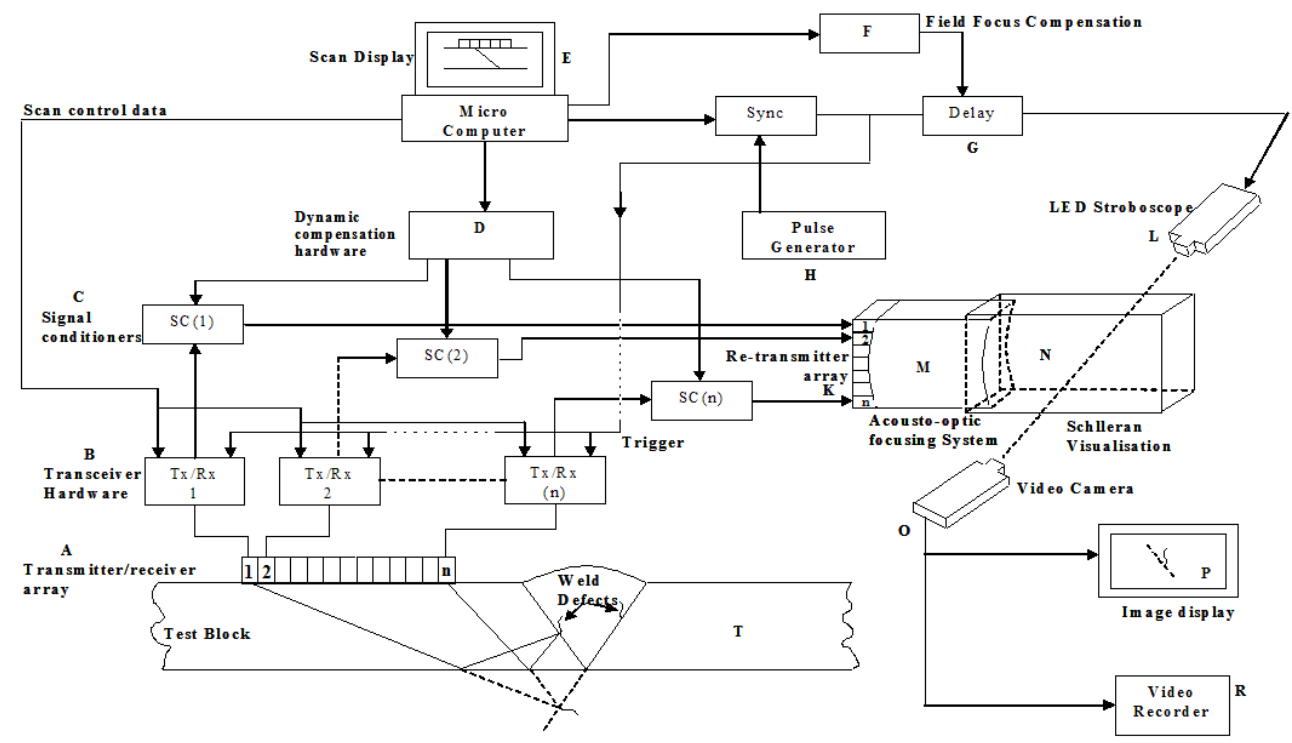

Figure 14. A hybrid imaging Topology with computer controlled scanning and acoustooptical image reconstruction (Expanded block diagram)

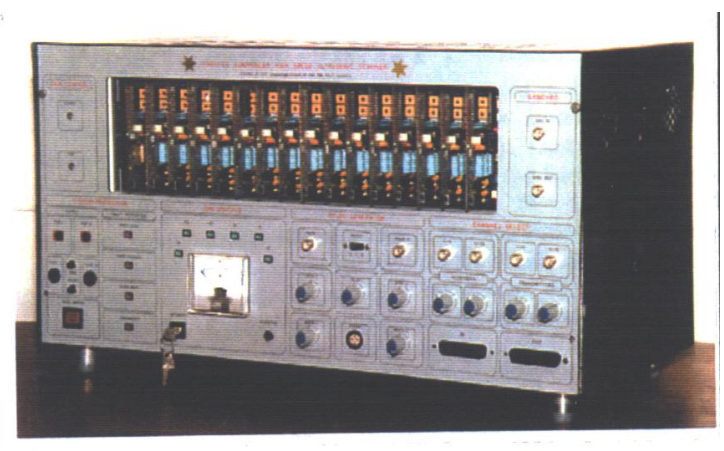

Figure 15. The first prototype of the hybrid scanner (GB2278443B)

Since the principle of acousto-optical imaging is very different to that of the conventional methods, some of the requirements of electronics and ultrasonic hardware is very different to that of the conventional systems in many ways. Since the received signals are converted back into ultrasound signals and retransmitted into an acoustic modulator to produce optical effects, 
the technique relies on the preservation of amplitude and phase characteristics of the signals to a high degree. Also, adequate signal power is required to produce acousto-optical modulation in the image medium to make the acoustic map of the object field visible.

For the first hybrid prototype system, the excitation pulses generated were $2 \mathrm{MHz}$ single sinusoidal pulses of the order of $120 \mathrm{Vpp}$ as shown in Figure 16 below. These pulses were shaped to obtain the near-ideal response from the transducer elements.

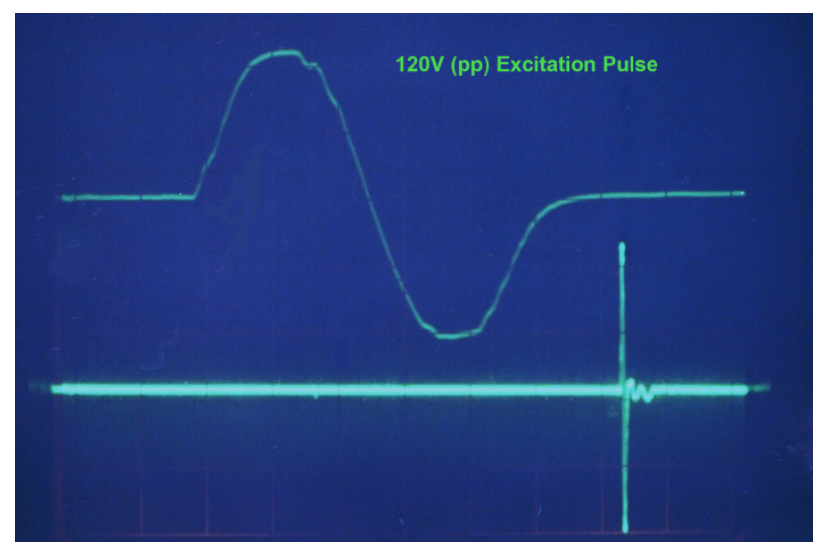

Figure 16. Single sinusoidal excitation pulse

The front-end receiving section of the hardware consists of wideband amplifiers $(15 \mathrm{MHz})$ with a variable gain of up to $60 \mathrm{~dB}$. The output impedance is of the order of $50 \mathrm{Ohms}$ with a maximum output swing capability of $120 \mathrm{~V}$ pp. Input surge protection up to $1 \mathrm{kV}$ was provided with a very low recovery time to achieve minimal dead-zone. Although the above prototype scanner was equipped with 30 channels, only 8 channels were used for the feasibility study. This represented just $1.6 \mathrm{~cm}$ acoustic aperture. No dynamic compensation was used. However, the performance as can be seen from statically scanned images was still very good.

\subsubsection{Operational modes}

The system may be operated in the axial mode for very high-speed imaging or be used in the scanning mode to cover a wide field. In the coaxial mode, the system resembles in operation to that shown in Figure 10 -12, producing narrow-field linear array B-mode images covered by the aperture of the array. In the scanning mode the system is under the control of the computer and can be used to produce wide-field, high resolution B-mode sector scan images, or be programmed to scan along any specific areas of the target. In order to achieve field uniformity for off-axis imaging, scan-angle derived field-focus compensation (FFC) and dynamic compensation (DCH) may be applied. In the scanning mode, wideband insonifying pulses are beamed to target areas by phased array beam steering. However, when receiving the echoes from the targets, there is no requirement for beam forming at all, as the system 
essentially behaving as an active, acousto-optical imaging device operating in real time. Therefore, even in the scanning mode, this allows very high temporal resolution to be achieved since one beam produces a complete image field or image zone. Furthermore, the whole of the effective aperture is utilized for each image frame and therefore diffraction-limited lateral resolution approaches the theoretical maximum.

If the scanning angles are small, the system could produce images without the need for any compensation. This is the simplest mode of scanning operation. For larger beam angles in the scanning mode, compensation to account for the reduction in sensitivity and field uniformity may be applied by the control of insonifying energy, receiver gain and scan derived electronic phase delays, which is an area for further development.

\subsection{Results from the $1^{\text {st }}$ hybrid scanner prototype}

As mentioned above, although the prototype was equipped with 30 channels, the number of channels used for the feasibility study was just 8 with an effective aperture of only $1.6 \mathrm{~cm}$ (as marked on the receiving array in Figure 17a). This was because of a problem of some of the channels breaking into oscillations due to excessive capacitive feedback at the time of experiments. Nonetheless, it can be seen from Figure 17 that the images produced are still very good for the aperture used.

As mentioned above, in the coaxial mode, the system resembles in operation to that shown in Figure 10 to 12. The speed of imaging is very high, e.g. in a test block of steel $40 \mathrm{~cm}$ deep, a complete image is formed within $0.15 \mathrm{~ms}$ and the frame rate can be as high as $1 \mathrm{kHz}$ or more. Estimation of resolution capabilities previously obtained using the coaxial system of Figure 9 showed that the images can be produced to within one wavelength resolution in the axial direction and about 1.5 wavelengths laterally at about $60 \mathrm{~mm}$ below the surface of the test block; thus approaching theoretical limits.

In order to test the operation in the scanning mode; a test block with side drilled holes covering a wide sector was prepared as shown in Figure 17(a). Figure 17 (b), (c) \& (d) shows the images when the insonifying beam was steered statically (i.e. in manually selected angles) to image the 3 holes in the left, two in the centre and the two on the right-hand side of the test block.

Dynamic scanning was also verified. However, since the scan sector required for the above test object was very large $\left(\sim 120^{\circ}\right)$ peripheral geometrical aberration was significant. When statically scanned, aberration can be controlled for each angle manually by selecting stroboscopic firing delays to achieve best focus. For smaller sectors (e.g. $<60^{\circ}$ ) aberration was not a significant factor.

Although the prototype system was designed for NDT applications, attempts to image biological moving tissues, such as heart valves, revealed excellent temporal resolution as expected. However, since the transducer arrays used did not have the required element spacing characteristics and matching properties, and the sonoptics of the prototype was designed for industrial material, the prototype could not obviously produce true B-mode images for biological tissues although the potential for advancement in this area was clearly evident. 


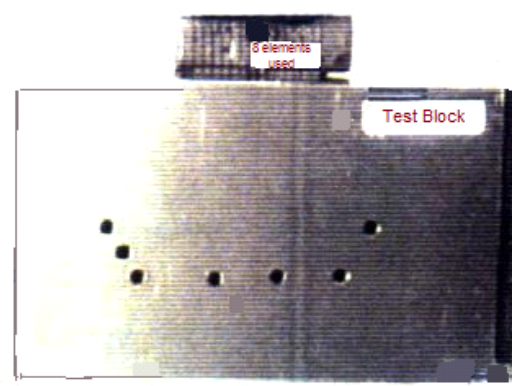

(a)

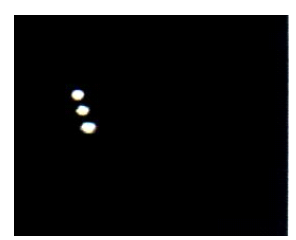

(b)

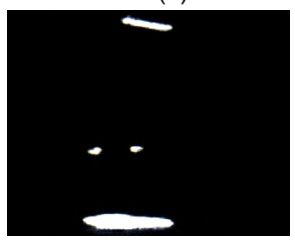

(c)

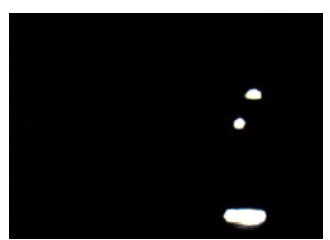

(d)

Figure 17. a) - Test Block. Statically scanned images of the test block

\section{Discussion - Breaking through the speed barrier}

In contrast to conventional line-serial computer based image reconstruction as used in existing systems, it has been demonstrated that the acousto-optical hybrid scanner has the potential to provide a number of important properties. These are:

- Ultimate speed of imaging as the image reconstruction is done using acousto-optical means thus "Breaking through the speed barrier" of the conventional line-serial imaging technology.

- In axial imaging mode, a complete image frame can be produced using a single ultrasonic pulse within its time of flight thus allowing ultimate imaging speed thus eliminating temporal artefacts when rapidly moving targets were imaged.

- In sector scanning mode image zones for each scan angle is produced allowing much higher frame-rates to be achieved, since very few scan angles are required as opposed to line serial scanning in conventional technology.

- A focused image of the whole object field is produced at the same instant of time without the need of any dynamic focusing as in the conventional technology.

- High lateral resolution, close to theoretical limits is achieved as the whole transducer aperture is used for each image frame.

As evident from the images, the signal-to-noise ratio (SNR) of the above prototype is very high. One of the reasons for this arises from the fact that true signals are coherent and the noise signals are not. Since the image reconstruction is based on amplitude and phase of signals, this 
allows true signals from targets to be coherently re-constructed in the image medium while the noise and out of phase signals are being largely rejected as evident from Figure 18 below. However, just like all ultrasound equipment multiple reflections between closely spaced targets could still cause artefacts. Therefore, inclusion of selective insonification of the object medium as in item No. 2 above should enable the system to achieve even higher SNR while suppressing artefacts when imaging near-field targets.

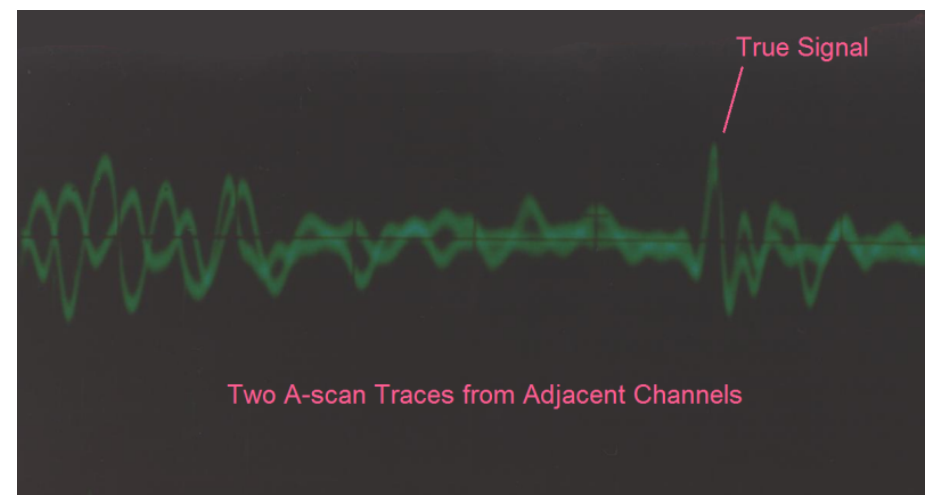

Figure 18. Noise suppression

Another important factor as mentioned in section 2 for any imaging technique is the dynamic range. In this respect, one of the typical bottlenecks is the limited dynamic range of display equipment. The present development can provide a greater display dynamic range when images are viewed with the naked eye as the acousto-optical modulator can provide a higher display dynamic range than images captured by a camera and presented on a typical conventional VDU.

\section{Conclusions}

Conventional ultrasonic imaging systems have inherent limitations such as low speed leading to temporal artefacts and in some cases limited lateral resolution; these being the result of lineserial scanning, lengthy processing and other limitations arising from the particular techniques used. The extent to which the above deficiencies affect performance has been analytically investigated.

In this respect, it has been shown that an alternative hybrid approach to imaging using acoustooptical image reconstruction could give clear advantages; reaching theoretical limits of performance in speed and resolution unachievable with the existing methods. This is mainly due to the combination of electronic and sonoptical image reconstruction, avoiding line-serial scanning and lengthy processing required by the conventional systems. The hybrid system reaches almost ultimate speed and resolution in the coaxial imaging mode. In the B-scan mode, 
it produces sector images formed by overlapping zones, but each zone requiring only one pulse to produce a complete zone image, thus gaining by far the highest speed of sector scanning compared to conventional methods.

Furthermore, the clarity and contrast of the images are high as the stroboscopic image mapping results in gating out many artefacts, problems due to reverberations and noise, to a high degree. Since the image reconstruction, by design, is based on coherent summation of signals in amplitude and phase corresponding to true targets satisfying the sonoptical focusing requirements, random noise gets suppressed without the need for further processing.

\subsection{Future work}

The typical results shown with the hybrid imaging system is applicable at present only to structural NDT for which it was designed. In order to produce true B-mode images for medical applications requires further work on the design of transducers and the sonoptical geometry to satisfy the conditions necessary for body tissue imaging, while accommodating the required dynamic range. In particular, it should be noted that the technique of image reconstruction is based on amplitude and phase integrity of signals; thus the inter-element uniformity of transducer elements and channels are crucial. In the case of medical imaging, the transducer element spacing is much smaller compared to NDT applications for any given frequency. For the above NDT system, the arrays were constructed using ordinary PZT transducer material. This is unlikely to be satisfactory in the case of medical transducers utilizing the above imaging techniques, since the requirements of transducer specifications are different and much tighter compared to conventional imaging to achieve good performance. Hence, transducers with piezo-composites or PVDF material may have to be developed for this application. Although the present system is designed to produce imaging in 2-D, extension of this technology to 3-D imaging in real-time is a distinct possibility which needs to be explored. This work and the development of dynamic compensation hardware will be taken forward in the next phase of development.

\section{Appendix}

\section{Prototype design considerations}

Since the active imaging system depicted in Figure 5 in section 4 above is required to produce a direct acoustic image which is made visible by synchronised stroboscopic light (Schlieren or other acoustooptical modulation technique), the design parameters for NDT applications were determined both by theoretical simulations and experimentation. For the construction of the transducer arrays, bearing in mind the tight requirements for elemental uniformity, number of parameters for a selected centre frequency of $2 \mathrm{MHz}$ were determined which included [15]:

- Estimation of number of channels (30 for the initial design)

- Transducer element spacing (2 $\mathrm{mm}$ for the initial design) 
- Element width-to-gap ratio

- Physical size, shape and transducer material

The primary aim was to obtain an idea of the sharpness of the images and the extent of artefacts that may be formed in a given image space. Since the transducer backings were conductive, maintaining a low level of electrical cross-coupling was required for the size of the backing thus the element gap spacing was kept around $0.3 \mathrm{~mm}$. For visualisation of the acoustic image formed in the modulating medium, the requirements for channel characteristics were then determined. These included practical determinations of number of parameters including:

- Typical and worst case input signal levels

- Output signal power required from each channel

- Phase linearity and bandwidth considerations

- Signal dynamic range

- Input and output impedances

- Element damping characteristics

\section{Acoustooptical design considerations}

As mentioned in section 4 above, as opposed to conventional image reconstruction, the passive DUVD system had some ideal properties for ultrasonic imaging namely, image linearity and isochronicity. Maintaining the same properties in the active system was therefore important. The requirement for image linearity as determined by Hansted [12] for the DUVD is given below.

\section{Maintaining image linearity}

Figure 19 shows the two-lens system of the DUVD.

Using paraxial ray analysis, it can be shown that in order to maintain image linearity throughout the image field, the only requirement is to make the focal point of the two acoustic lenses coincident. This however changes the lateral magnification as given in equation 10 , but since this is a constant it does not affect linearity or image quality and can be easily compensated if necessary.

\section{Maintaining isochronicity}

As stated in section 4 above, iscochronicity means that when the test object is insonified with a short acoustic pulse, all the echoes from the different targets, irrespective of their special distribution within the object field, arrive simultaneously at their respective image points thus giving the system its ability to display the whole image at once.

The above capability is in stark contrast to the need for line serial scanning in conventional imaging, giving the system its speed - the highest theoretically possible speed of imaging as it 


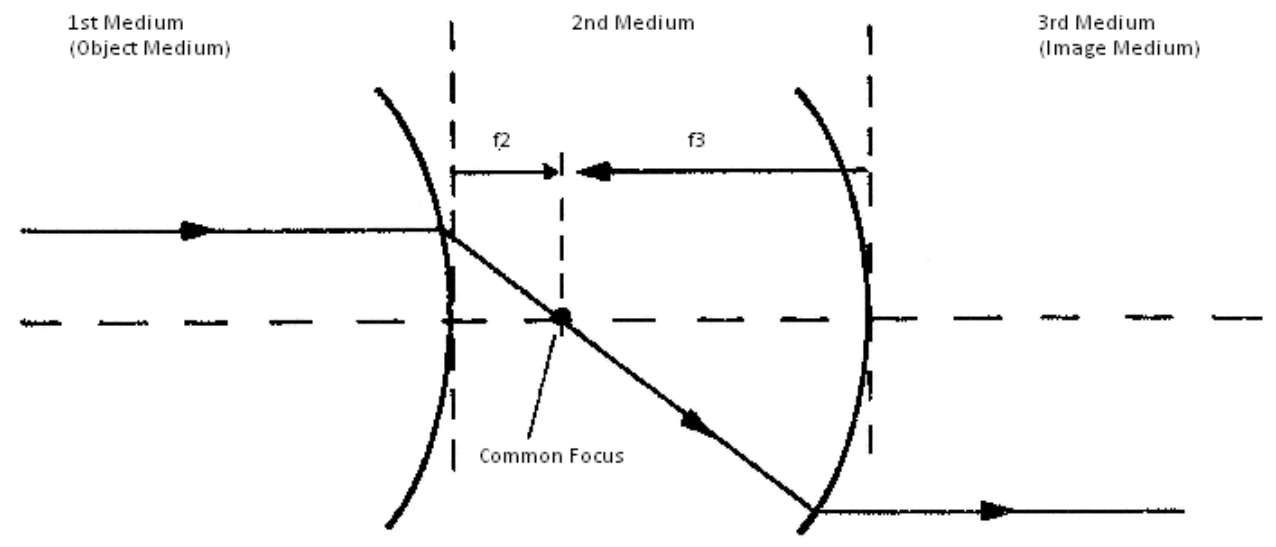

Figure 19. Maintaining image linearity

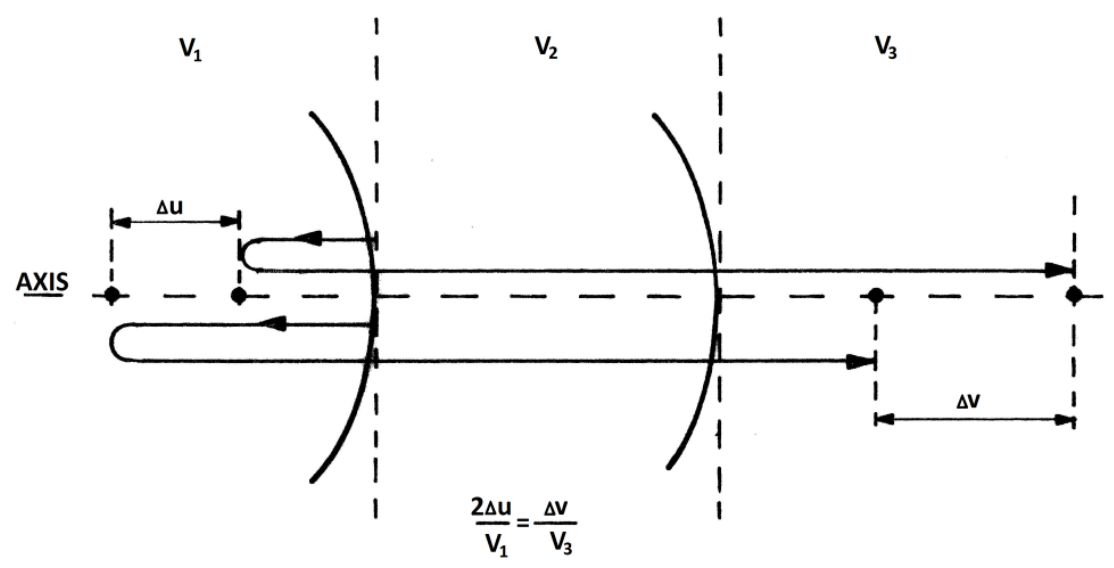

Figure 20. Requirements for maintaining Isochronicity

produces images with a single pulse practically within the time of flight of the pulse echoes in the object and image media. The conditions required for iscochronicity as determined by Hansted for his DUVD system with reference to Figure 20 is given below.

It can be clearly seen that the necessary and sufficient condition for the images of two objects in the object medium with velocity $\mathrm{v}_{1}$ to be brought to focus at the same instant of time in the image medium with velocity $\mathrm{v}_{2}$ could be given as: 


$$
\frac{2 \Delta u}{V_{1}}=\frac{\Delta v}{V_{3}}
$$

where, $\Delta \mathrm{u}$ is the axial separation of the objects in the object medium and $\Delta \mathrm{v}$ is the axial separation of the respective image points in the image medium. In terms of respective focal lengths in object and image media $\left(f_{1} \& f_{4}\right)$ the condition depicted in equation (6) is achieved for this lens combination with a common focus in the medium $v_{2}$ when $\mathrm{f}_{1} \& \mathrm{f}_{4}$ are related such that:

$$
\frac{f_{1}}{f_{4}}=\frac{1}{\sqrt{2}}
$$

Using paraxial ray analysis, it can also be shown that when satisfying the above necessary conditions, the maximum object distance $\left(\mathrm{U}_{\max }\right)$ and maximum image distance $\left(\mathrm{v}_{\max }\right)$ can be written as:

$$
\begin{aligned}
& U_{\text {max }}=\left(\frac{f_{1} f_{2}}{f_{3}}\right)+f_{1} \\
& v_{\text {max }}=\left(\frac{f_{3} f_{4}}{f_{2}}\right)+f_{4}
\end{aligned}
$$

where, $f_{1} \& f_{2}$ are the focal lengths of the first lens defined by medium velocities $v_{1} v_{2}$, and $f_{3}$ \& $\mathrm{f}_{4}$ are that determined by the $2^{\text {nd }}$ acoustic lens.

Thus the necessary and sufficient conditions for linearity and Isochronicity for the acoustic imaging system are:

- The internal focal points must be coincident

- The external focal lengths must be related in accordance with that given in equation (7)

Using paraxial ray analysis taking also into account the conditions necessary for Isochronicity, it can be shown that there is however lateral image magnification (as mentioned above) such that:

$$
M_{A}=\sqrt{2} M_{L}
$$

Where, $M_{A}$ and $M_{L}$ are axial and lateral magnifications respectively. This does not affect image linearity and can be compensated if necessary. 


\section{Acknowledgements}

Author wishes to express his sincere thanks to the School of Engineering of the Robert Gordon University, UK where much of the developmental work was carried out and to Mr Andrew Fairhead, ultrasound engineer of the Aberdeen Royal Infirmary, NHS Grampian, for his valuable comments and advice regarding medical imaging systems.

\section{Author details}

G. P. P. Gunarathne

School of Engineering, Robert Gordon University, Aberdeen, UK

\section{References}

[1] http://inventors.about.com/od/sstartinventions/a/sonar_history.htm (Accessed 24th February 2013).

[2] http://www.ob-ultrasound.net/history1.html (Accessed 24th February 2013).

[3] http://www.ndt-ed.org/EducationResources/CommunityCollege/Ultrasonics/Introduction/history.htm (Accessed 1st Jan 2013).

[4] Three-dimensional imaging system: Olaf von Ramm and Stephen Smith, Duke University, USA, United States Patent - 4694434 http://www.freepatentsonline.com/ 4694434.html (Accessed 1 ${ }^{\text {st }}$ Jan 2013).

[5] Portable PC-based Ultrasound scanners, Imaging Tools, Telemed http://www.hospitalmanagement.net/contractors/imaging/telemed/ visited $1^{\text {st }}$ Jan 2013.

[6] Zone Sonography, Zonare Medical systems, http://cdn.zonare.com/ 0d87056c5a4bae2587f9710a5d669d33.pdf (Accessed 24th February 2013).

[7] Coded Excitation: GE Healthcare, http://www.gehealthcare.com/usen/ultrasound/ education/products/techcodex.html (Accessed 1st Jan 2013)

[8] Digitally Encoded Ultrasound: GE Healthcare, http://www.gehealthcare.com/usen/ ultrasound/education/products/techedu.html (Accessed 1st Jan 2013)

[9] Research Corporation Technologies, "Tissue Harmonic Imaging": http://rctech.com/ resources/downloads/THI_Overview_9-06.pdf visited 1 1' Jan 2013.

[10] Ultrasound C-can camera: Acoustocam, http://www.reinforcedplastics.com/view/ 1180/ultrasound-camera-makes-internal-composite-damage-easy-to-find/ (Visited $2^{\text {nd }}$ Jan. 2013). 
[11] G.P.P. Gunarathne, “Ultrasonics Imaging, Invited Presentation at the World Congress of Engineering 2007, London, UK, July 2007.

[12] P.D. Hanstead: "Three Dimentional Imaging of Ultrasound - Direct Ultrasonic Visualisation of Defects", Nature 239, 1972, pp 273-274.

[13] A.J. Hayman: "Schlieren Visualisation of Ultrasonic Images", PhD Thesis, City University, London, 1977.

[14] Y. Bar-Cohen, B. Ben-Joseph and E. Harnick: "Compact Sensitive Instrument for Direct Visualisation of Defects", Rev. Sci. Instruments, Vol.49, 1978, pp 1709-1911.

[15] G.P.P. Gunarathne and J Szilard:"A Real-time High Frame Rate Ultrasonic Imaging System", IEEE Ultrasonics Symposium, San Francisco, USA, Conference Proceedings, 1985, pp 98-203.

[16] G.P.P. Gunarathne and J Szilard: "A New stroboscope for Schileren and Photoelastic Visualisation of Ultrasound", Ultrasonics, July 1983, pp 188-190.

[17] G.P.P. Gunarathne: "A new real-time Ultrasonic Imaging System" IEE International Conference on Acoustic Sensing and Imaging, Conference Publication No. 369, pp93 - 98, March 1993.

[18] G.P.P. Gunarathne: “A Real-time, Hybrid Ultrasonic Display/Imaging System for Medical and Industrial Applications", United Kingdom Patent: GB2278443B, July1997.

[19] G.P.P. Gunarathne: "Real-time Ultrasonic Imaging and Advancements in Non- conventional Methods" ${ }^{2}$ MTC 2008 - IEEE International Instrumentation and Measurement Technology Conference, Victoria, Vancouver Island, Canada, May 12-15, 2008. 
